\title{
Ramo Dorsal del Nervio Ulnar: un Aporte Significativo a la Inervación Sensitiva del Dorso de la Mano
}

\author{
Dorsal Branch of the Ulnar Nerve: a Significant Contribution \\ to the Sensory Innervation of the Dorsum of the Hand
}

\author{
Tiznado, G.*; Olave, E.**; del Sol, M. ${ }^{* * * * * *} \&$ Sousa-Rodrigues, C. ${ }^{* * * * *}$
}

TIZNADO, G.; OLAVE, E.; DEL SOL, M. \& SOUSA-RODRIGUES, C. Ramo dorsal del nervio ulnar: un aporte significativo a la inervación sensitiva del dorso de la mano. Int. J. Morphol., 31(4):1162-1167, 2013.

RESUMEN: La inervación sensitiva del dorso de la mano está dada principalmente por ramos provenientes del ramo superficial del nervio radial (RSNR) y del ramo dorsal del nervio ulnar (RDNU). La distribución del primero, abarcaría la zona desde el pulgar a la mitad radial del dedo anular y el segundo, la mitad ulnar de este dedo así como el dedo mínimo. El presente estudio tuvo por finalidad describir el origen, trayecto, distribución y ramificación del ramo dorsal del nervio ulnar. El estudio mediante disección fue realizado en 30 miembros superiores de individuos adultos, Brasileños y 6 miembros superiores de individuos adultos, Chilenos, fijados en formaldehido al $10 \%$, de los cuales 16 eran miembros del lado derecho y 20 del lado izquierdo. Los primeros estaban localizados en la Universidade Estadual de Ciencias da Saúde de Alagoas, Maceió, Brasil y los segundos en la Facultad de Medicina de la Universidad de la Frontera, Temuco, Chile. El RDNU se originó a nivel del tercio distal del antebrazo proximal al proceso estiloides ulnar en 34 miembros (94,4\%). En todas las muestras la distribución del RDNU en el dorso de la mano se comporta de forma similar con la diferencia de tener o no un ramo comunicante con ramos del RSNR. De acuerdo a esto clasificamos su disposición en un grupo con ramo comunicante y en otro sin ramo comunicante. Se observó 21 muestras $(58,3 \%)$ con ramo comunicante y 13 sin el mismo. El comportamiento de las divisiones del ramo dorsal del nervio ulnar es descrito y padronizado. En los 21 casos el RDNU proporcionó 5 nervios digitales, correspondientes al dedo mínimo, anular y el de la parte ulnar del dedo medio. Sin embargo, en estos casos el RDNU recibió colaboración de fibras provenientes de RSNR. En 13 casos (36,1\%) el RDNU proporcionó 5 nervios digitales dorsales, correspondientes al dedo mínimo, anular y la parte ulnar del dedo medio, sin colaboración del RSNR. En 2 casos $(5,6 \%)$ todos los nervios digitales dorsales fueron proporcionados por el RSNR, sin aportes del RDNU. En relación a la presencia de comunicación entre el RDNU y el RSNR se observó que las fibras componentes se originaron desde el RSNR y finalizaron en el ramo lateral del RDNU. La disposición del RDNU no posee gran variación en su distribución y tiene un aporte significativo en la inervación sensitiva del dorso de la mano.

PALABRAS CLAVE: Anatomía; Mano; Inervación sensitiva; Ramo dorsal del nervio ulnar.

\section{INTRODUCCIÓN}

La inervación sensitiva del dorso de la mano está dada principalmente por ramos provenientes del ramo superficial del nervio radial y del ramo dorsal del nervio ulnar. La distribución del primero, abarcaría la zona desde el pulgar a la mitad radial del dedo anular y el segundo, la mitad ulnar de este dedo así como el dedo mínimo (Williams et al., 1995). También se ha descrito que la distribución del primero comprendería la zona desde el pulgar a la mitad radial del dedo medio y el segundo, desde la mitad ulnar de este dedo hasta el dedo mínimo (Rouvière \& Delmas, 2005). La literatura refiere varios autores que se han referido sólo a los ramos que derivan del ramo superficial del nervio radial, pero otros, como Botte et al. (1990), Casoli et al. (2004), Goto et al. (2010) y Cavusoglu et al. (2010) han estudiado y descrito el recorrido realizado por el ramo dorsal del nervio ulnar.

Debido al hecho de que los tratados clásicos y ulteriores estudios anatómicos no presentan descripciones detalladas de la topografía de los ramos sensitivos en el dorso de la mano, el presente estudio tiene por finalidad describir el origen, trayecto, distribución y ramificación del ramo dorsal del nervio ulnar, con el fin de proveer información sobre

\footnotetext{
* Universidad San Sebastián, Sede Osorno, Chile.

** Facultad de Medicina, Universidad de La Frontera, Chile.

*** Centro de Investigación en Ciencias Biomédicas, Universidad Autónoma de Chile.

**** Universidade de Ciencias da Saúde de Alagoas; Universidade Federal de Alagoas, Maceió, Brasil.
} 
su distribución y respectivas variaciones, contribuyendo a complementar los conocimientos ya conocidos, de utilidad, tanto en la clínica, como en los procedimientos quirúrgicos que se realizan en el dorso de la mano.

\section{MATERIAL Y METODO}

El estudio fue realizado en 30 miembros superiores de individuos adultos, Brasileños y 6 miembros superiores de individuos adultos, Chilenos, fijados en solución acuosa de formaldehido al $10 \%$, de los cuales 16 eran miembros del lado derecho y 20 del lado izquierdo. Los primeros estaban localizados en los Laboratorios de Anatomía de la Universidade Estadual de Ciencias da Saúde de Alagoas, Maceió, Brasil y los segundos en los Laboratorios de Anatomía de la Facultad de Medicina de la Universidad de la Frontera, Temuco, Chile.

Se efectuó la disección del dorso de cada una de las manos, con ayuda de una lupa Ranson con aumento de $3 \mathrm{x}$ para facilitar la identificación de estructuras finas.

Se trazó una incisión horizontal en el dorso de la muñeca. Desde la parte mediana de la incisión horizontal, se llevo a cabo una incisión hacia distal que llegó hasta la falange intermedia del dedo medio y otra incisión proximal que se dirigió hacia el dorso del antebrazo. Por el margen lateral y medial de la mano, desde la incisión horizontal en la muñeca, hasta el margen lateral del dedo mínimo y pulgar, se hicieron las otras incisiones.

Se retiró la piel y el tejido adiposo subcutáneo, evidenciando los ramos sensitivos del dorso de la mano: ramo superficial del nervio radial (RSNR) y el ramo dorsal del nervio ulnar (RDNU), además de estructuras vecinas a éstos.

El proceso estiloides de la ulna (PEU) fue considerado como punto de referencia, para realizar las siguientes mediciones: -Distancia entre el origen del RDNU y el PEU, - Distancia en que el RDNU se hace superficial y el PEU, - Distancia entre el PEU y la división del RDNU en ramos ulnar y radial.

Las mediciones se realizaron con un caliper digital marca Mitutoyo de 0,05 mm de precisión. Todas las muestras fueron fotografiadas y esquematizadas con una cámara digital Panasonic Lumix ZS8 de 14,1 mega pixeles marca Panasonic. Se utilizó estadística descriptiva para los valores promedio y desviación estándar, empleando para ello el programa Microsoft Excel 2010.

\section{RESULTADOS}

El RDNU se originó a nivel del tercio distal del antebrazo proximal al PEU en 34 miembros $(94,4 \%)$, originándose en promedio a $80,9 \pm 25,7 \mathrm{~mm}$ proximal al PEU, continuando por la profundidad del músculo flexor ulnar del carpo (FUC), para emerger, también proximal al PEU, por entre la ulna y el FUC. A este nivel perforó la fascia profunda para hacerse subcutáneo y continuar un trayecto hacia la parte medial del dorso de la mano. El nivel donde se hizo superficial se localizó a una distancia promedio de 37,1 \pm 9,5 mm proximal al PEU.

Luego, el RDNU se dividió en un ramo ulnar y un ramo radial, punto de división localizado a un promedio de 22,3 \pm 12,2 mm proximal al PEU. El ramo ulnar siguió un trayecto palmar al PEU y se dirigió hacia la parte medial del carpo y el quinto metacarpiano, formando distalmente el nervio digital dorsal ulnar del dedo mínimo (C5). El ramo radial continuó su recorrido hacia el dorso de la mano, pasando medial y palmar al PEU en todos los casos, dividiéndose distalmente a este último, en un ramo medial y otro lateral, que se distribuyeron inervando sensitivamente la porción medial del dorso de la mano y la porción proximal del quinto, cuarto y tercer dígito.

En 2 miembros el RDNU se encontraba ausente y la inervación del dorso de la mano fue realizada completamente por el RSNR.

En todas las muestras la distribución del RDNU en el dorso de la mano se comporta de forma similar con la diferencia de tener o no un ramo comunicante con ramos del RSNR. De acuerdo a esto clasificamos su disposición en un grupo con ramo comunicante y en otro sin ramo comunicante.

En el primer grupo, donde existe un ramo comunicante (Fig.1) hubo 21 muestras con esta característica y el RDNU se comportó de la siguiente manera:

En la parte dorsomedial de la mano el RDNU se dividió proximal al PEU, en dos ramos, un ramo ulnar y un ramo radial. El ramo ulnar siguió un trayecto palmar y medial al PEU y continuó por el margen medial del quinto metacarpiano, para conformar, en la falange proximal del quinto dedo, el nervio digital dorsal ulnar del dedo mínimo (C5). En tanto, el ramo radial continuó hacia el dorso de la mano, pasando siempre palmar al PEU, dividiéndose en 20 casos distal al PEU y en el caso restante, proximal al mismo, en un ramo medial y uno lateral. El ramo medial se dirigió hacia el cuarto espacio interóseo donde se dividió, 
formando el nervio digital dorsal radial del dedo mínimo (R5) y el nervio digital dorsal ulnar del dedo anular (C4). Esta división se produjo distal al PEU. Mientras que el ramo lateral tomó una dirección oblicua hacia el tercer espacio interóseo, dividiéndose distal al PEU, en el nervio digital dorsal radial del dedo anular (R4) y el nervio digital dorsal ulnar del dedo medio (C3). En estos casos, el ramo ulnar del ramo dorsal del
RSNR emitió un ramo comunicante (RC), que se originó distal al PER. Éste siguió un recorrido distal, en sentido oblicuo hacia el tercer espacio interóseo, donde se comunicó con el ramo lateral del RDNU, en un punto distal al PEU.

En el segundo grupo (Fig.2), en el que observamos 13 muestras, la distribución del RDNU tiene características

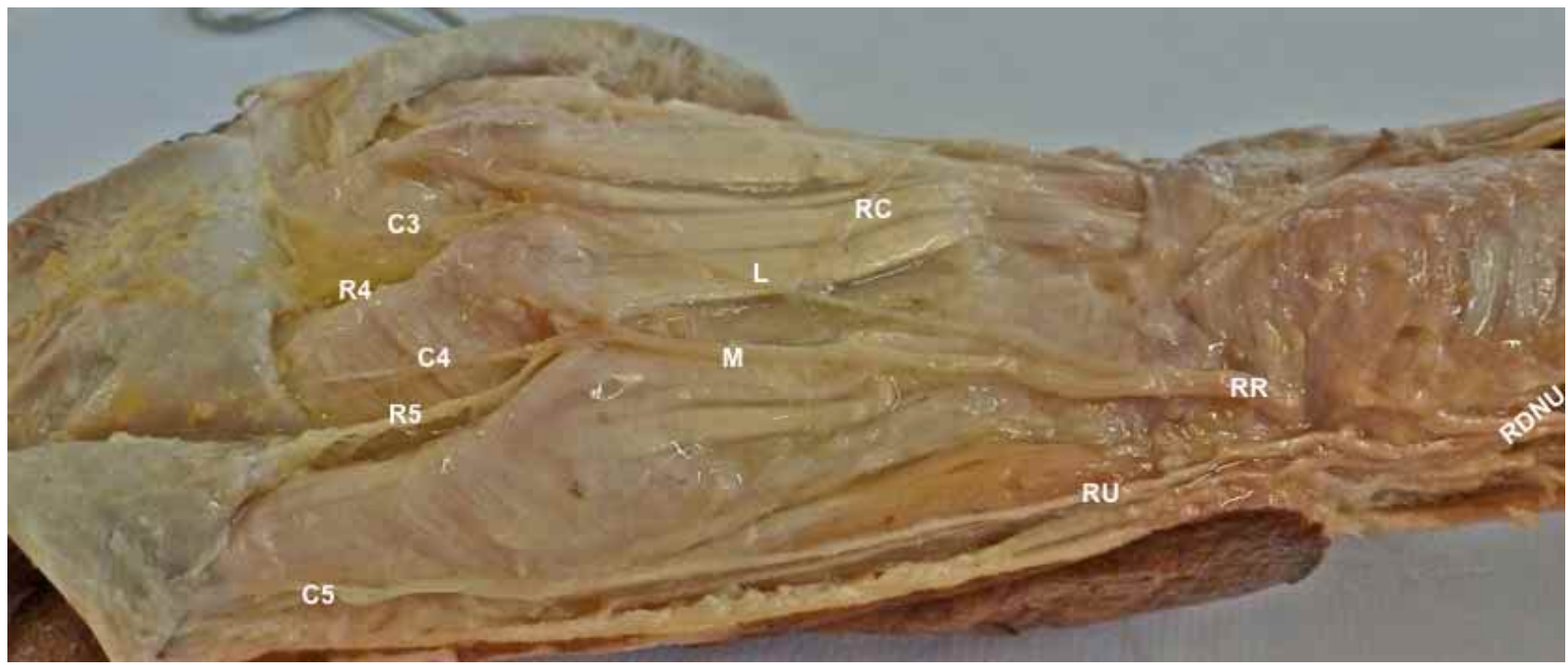

Fig. 1.- Aspecto ulnar de una mano izquierda. Ramo dorsal del nervio ulnar (RDNU); ramo radial (RR); ramo ulnar (RU); ramo medial del ramo radial (M); ramo lateral del ramo radial (L); nervio digital dorsal ulnar del dedo meñique (C5); ramo comunicante (RC); nervio digital dorsal radial del dedo meñique (R5); nervio digital dorsal ulnar del dedo anular (C4); nervio digital dorsal radial del dedo anular (R4); nervio digital dorsal ulnar del dedo medio (C3).

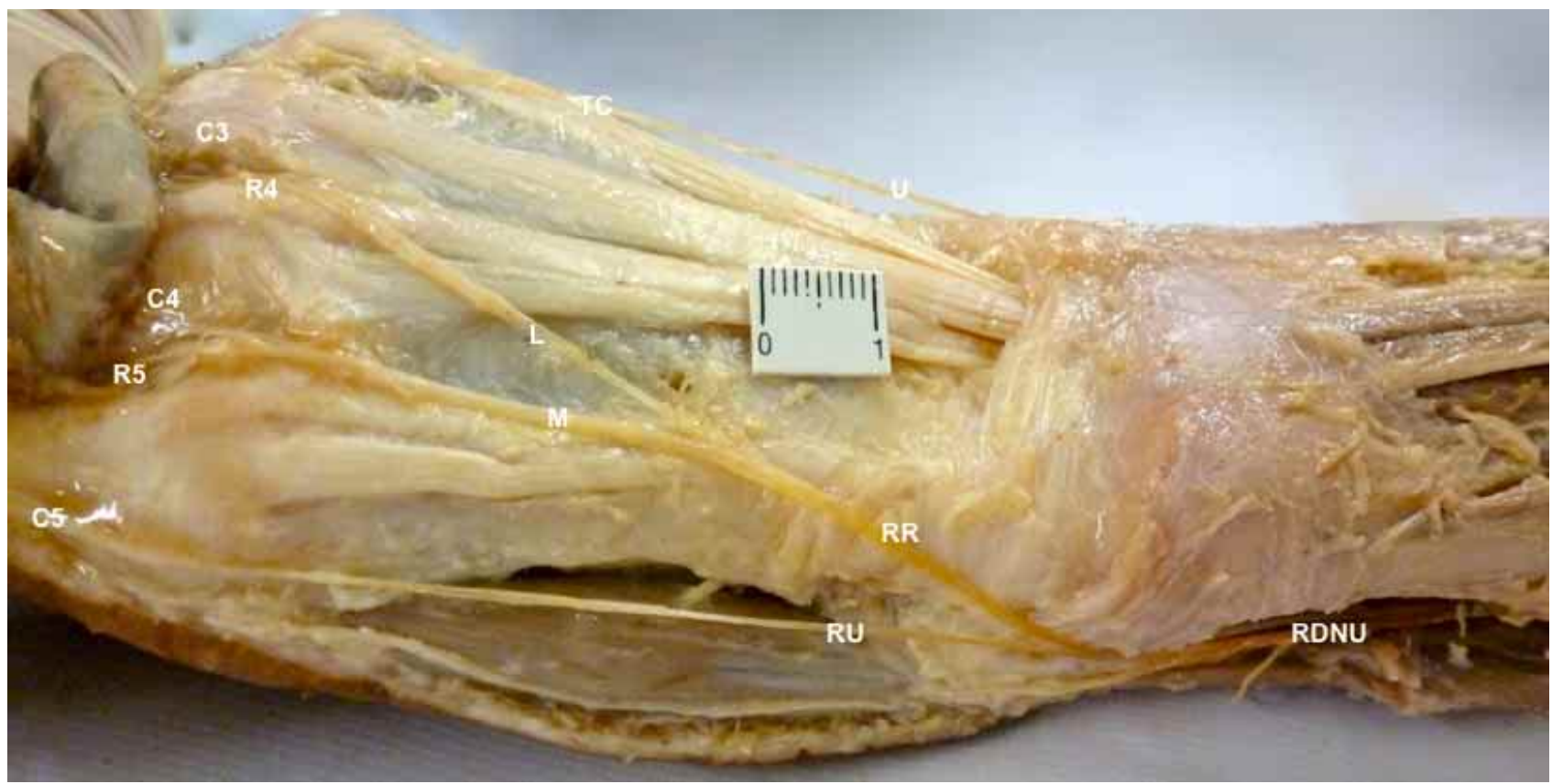

Fig. 2. Mano izquierda. Ramo dorsal del nervio ulnar (RDNU); ramo ulnar (RU); ramo radial (RR), ramo medial (M); ramo lateral (L), nervio digital dorsal ulnar del dedo meñique (C5), nervio digital dorsal radial del meñique (R5), nervio digital dorsal ulnar del dedo anular (C4); nervio digital dorsal radial del dedo anular (R4); nervio digital dorsal ulnar del dedo medio (C3); ramo ulnar del RSNR (U); tronco común (TC). 
similares, con la diferencia que no existe un aporte de fibras, representado por un ramo comunicante, desde el RSNR. Una disposición especial se observó en dos miembros $(5,56 \%)$, de cadáveres, nacionalidades y de lados distintos. En estos casos se observó que la inervación sensitiva del dorso de la mano fue proporcionada exclusivamente por el RSNR, sin presencia de ramos del nervio ulnar.

Con respecto al número de nervios digitales dorsales proporcionados por el RDNU se observó lo siguiente:

En 21 casos $(58,3 \%)$ El RDNU proporcionó 5 nervios digitales, correspondientes al dedo mínimo, anular y el de la parte ulnar del dedo medio. Sin embargo, en estos casos el RDNU recibió colaboración de fibras provenientes de RSNR.

En 13 casos $(36,1 \%)$ el RDNU proporcionó 5 nervios digitales dorsales, correspondientes al dedo mínimo, anular y la parte ulnar del dedo medio, sin colaboración del RSNR.

En 2 casos $(5,6 \%)$ todos los nervios digitales dorsales fueron proporcionados por el RSNR, sin aportes del RDNU.

En relación a la presencia de comunicación entre el RDNU y el RSNR se observó que hubo comunicación entre estos ramos en 21 casos $(58,3 \%)$. En estos casos, fueron fibras que se originaron desde el RSNR y finalizaron en el ramo lateral del RDNU.

\section{DISCUSION}

Desde el punto de vista de las mediciones realizadas en el RDNU, autores como Botte et al.; Casoli et al.; Cavusoglu et al., reportaron que el RDNU se originó a una distancia promedio de $8,3 \mathrm{~cm}, 8 \mathrm{~cm}$ y $8,36 \mathrm{~cm}$ respectivamente, considerando como punto de referencia el hueso pisiforme. Sin embargo, en nuestro estudio utilizando el proceso estiloides de la ulna (PEU) como punto de referencia, el RDNU se originó entre 52 y 162 mm (promedio 80,9 mm) proximal al PEU, coincidente con los autores mencionados, ya que nuestra medición se efectuó proximal al hueso pisiforme, con diferencia notable a lo observado por Goto et al. quienes hallaron que éste se originó en promedio a 34 mm proximal al PEU.

Otros autores a través de reportes de casos como Paul et al. (2006) y Lama et al.(2009) encontraron un origen alto del RDNU cercano al nivel del codo.
En nuestro estudio, el RDNU después de originarse se hizo superficial pasando por entre la ulna y el FUC, coincidiendo con lo relatado por numerosos autores (Goto et al; Testut \& Latarjet (1972); Chatain \& Bustamante (1986); Sinelnikov (1986); Williams et al; Moore \& Dalley (2002); Latarjet \& Ruiz Liard (2004); Llusá et al. (2004) ;Rouvière \& Delmas. En tanto, Botte et al. y Cavusoglu et al. registraron la distancia de este punto a un promedio de 50 y 54,7 $\mathrm{mm}$, respectivamente, proximal al hueso pisiforme, mientras que en nuestro estudio, relacionando este punto al PEU, la distancia promedio en que el ramo se hizo superficial fue de 37,1 mm proximal al PEU.

Tanto Casoli et al. como Cavusoglu et al. describieron la ramificación del RDNU, reportando en sus estudios que el RDNU se dividió en dos ramos, donde el ramo medial se dirigió hacia al margen medial de la zona del quinto metacarpiano, mientras que el ramo lateral se dividió distalmente en dos ramos que se dirigieron hacia la parte ulnar del dedo anular y a la parte radial del dedo mínimo. La división del RDNU coincide con lo observado en nuestro estudio, donde además determinamos que la división del RDNU se produjo en promedio a 22,3 mm proximal al PEU, en tanto que la división del ramo radial ocurrió a 26,8 mm distal a éste último, pero la distribución en nuestro estudio fue diferente. Sin embargo, Botte et al. reportaron que el RDNU proporcionó entre dos a tres ramos, sin describir la dirección seguida por cada uno.

La clásica descripción sobre la inervación sensitiva del dorso de la mano, es atribuida a ramos procedentes del nervio radial y ulnar, en que el primero se encarga de la inervación lateral del dorso de la mano y el segundo del aspecto medial del dorso de la mano, incluyendo las falanges proximales de los dedos. Al revisar los tratados clásicos de anatomía, se puede apreciar como diferentes autores tienen conceptos distintos del área que abarca cada uno de estos nervios. Por ejemplo Testut \& Latarjet; Chatain \& Bustamante; Sinelnikov; Williams et al.; Moore \& Dalley; Latarjet \& Ruiz Liard; Llusá et al.; Rouvière \& Delmas señalaron que el RSNR y el RDNU proporcionan el 50\% cada uno de los nervios digitales dorsales. Sin embargo, Ellis (2006) indicó que el RSNR proporciona los nervios digitales dorsales del 1er, 2do, 3er y el aspecto radial del 4to dedo, en tanto que el RDNU proporcionaría los nervios digitales dorsales del 5 to y aspecto ulnar del 4 to dedo. Por otro lado Leversedge et al.(2010) son más específicos al detallar que en el $45 \%$ de los casos, el RSNR inerva $21 / 2$ de los dedos radiales y en el $30 \%$ de los casos inerva los $31 / 2$ dedos radiales.

En el caso de nervios digitales dorsales provenientes del RDNU, son aún menos los estudios que describen detalladamente como ocurre esta situación. Los estudios lleva- 
TIZNADO, G.; OLAVE, E.; DEL SOL, M. \& SOUSA-RODRIGUES, C. Ramo dorsal del nervio ulnar: un aporte significativo a la inervación sensitiva del dorso de la mano Int. J. Morphol., 31(4):1162-1167, 2013.

dos a cabo por Casoli et al. y Cavusoglu et al. señalaron que en la totalidad de los casos, el ramo ulnar del RDNU proporciona únicamente el nervio digital dorsal ulnar del dedo mínimo. Igualmente, estos autores indicaron que en todos los casos, el ramo radial del RDNU conformó solamente el nervio digital dorsal radial del dedo mínimo y el nervio digital dorsal ulnar del dedo medio, situación que no fue observada en nuestro estudio, en el cual reportamos que el ramo radial del RDNU proporcionó los nervios digital dorsal ulnar del dedo anular y dedo medio, como también los nervios digital dorsal radial del dedo mínimo y anular.

En relación a la comunicación entre el RSNR y el RDNU, los tratados clásicos de anatomía dan por hecho la existencia de esta comunicación, sin embargo, esto no se ve reflejado en la mayoría de los estudios consultados. Por ejemplo Botte et al. reportaron una prevalencia de solo 3,8\%, mientras que Vergara-Amador \& Nieto (2010) refirieron no encontrar ningún tipo de conexión entre ambos ramos.

Por su parte, Loukas et al. (2008) llevaron a cabo un estudio muy detallado en 200 manos, donde estudiaron específicamente la conexión entre el RSNR y el RDNU, observando que esta situación se presentó en el $60 \%$ de los casos, dividiendo esta comunicación según sus características morfológicas, en cuatro tipos.

El tipo I fue el más prevalente y se observó en el $59,1 \%$ de los casos y consistía en un ramo comunicante que se originaba desde el RSNR y se dirigía distalmente hasta el RDNU. En el tipo II $(19,1 \%)$ el ramo comunicante se originó en el RDNU y se dirigió distalmente hasta el RSNR. En el tipo III (3,3\%) el ramo comunicante se dirigió perpendicularmente entre el RSNR y RDNU, por tanto era imposible determinar el punto de origen y en el tipo IV $(18,3 \%)$ hubo múltiples ramos comunicantes.

En nuestro estudio pudimos corroborar lo reportado por Loukas et al. al observar esta comunicación entre ambos ramos en el 58,3\% de las muestras. Las características de la comunicación observadas en todas las muestras, coinciden con el tipo I descrito por Loukas et al. Similar resultado fue lo que describió Zaki (2006), al encontrar comunicación entre el RSNR y el RDNU en el 65\% de los casos, sin embargo, no entrega detalles de las características del ramo comunicante. Por lo demás ninguno de los otros autores comoTellioglu et al. (2000); Bianchi (2002); Ikiz \& Uçerler (2004); Huanmanop et al.(2007); Klitscher et al. (2007); Robson et al. (2008); Tryfonidis et al. (2010); Samarakoon et al. (2011); Casoli et al.;Goto et al. y Cavusoglu et al. hicieron referencia a la existencia de la comunicación entre estos ramos. Se desconoce la existencia de estas diferencias tan notables, ya sea por tamaño de la muestra, variación individual de los cadáveres o método de detección.

Como se puede apreciar, el ramo dorsal del nervio ulnar tiene una distribución relativamente constante y tiene un aporte significativo en la inervación sensitiva del dorso de la mano humana.

TIZNADO, G.; OLAVE, E.; DEL SOL, M. \& SOUSA-RODRIGUES, C. Dorsal branch of the ulnar nerve: A significant contribution to the sensory innervation of the dorsum of the hand. Int. J. Morphol., 31(4):1162-1167, 2013.

SUMMARY: The sensory innervation of the dorsum of the hand is mainly given by branches from the superficial branch of the radial nerve (SBRN) and the dorsal branch of the ulnar nerve (DBUN). The distribution of the first, cover the area from the thumb to the radial half of the ring finger and the second, the ulnar half of this finger and the little finger. This study aimed to describe the origin, course, distribution and branches of DBUN. The study by dissection was performed in 30 upper limbs of adult Brazilian individuals and 6 upper limbs of adult Chilean individuals, fixed in 10\% formaldehyde, 16 of which were members of the right side and 20 on the left. The first individuals were located in the Universidade Estadual de Ciencias da Saude, Alagoas, Brazil and the second, in the Faculty of Medicine, Universidad de La Frontera, Chile. The DBUN originated at the distal third of the forearm proximal to the ulnar styloid process in 34 limbs (94.4\%).In all samples the DBUN distribution in the dorsum of the hand behaves similarly with the difference of having or not a communicating branch with the SBRN. Accordingly we classify in a group with communicating branch and another without communicating branch. 21 samples (58.3\%) with communicating branch and 13 (36.1\%) without it were observed. The division patterns of the dorsal branch of the ulnar nerve is described. In 21 cases the DBUN gave 5 digital nerves corresponding to the little finger, ring finger and the ulnar side of the middle finger. However, in these cases the DBUN received fibers from SBRN. In 13 cases (36.1\%) the DBUN gave 5 dorsal digital nerves, corresponding to the minimum finger, ring finger and the ulnar side of the middle finger, without collaboration of the SBRN. In 2 cases (5.6\%) the dorsal digital nerves were provided only by the SBRN without DBUN contributions. In relation to the presence of communication between DBUN and SBRN the component fibers were originated from SBRN and ended on the lateral branch of DBUN. The disposition of the DBUN has not large variation in distribution and has a significant contribution to the sensory innervation of the dorsum of the hand.

\section{KEY WORDS : Anatomy; Hand; Sensory innervation; Dorsal branch of ulnar nerve.}




\section{REFERENCIAS BIBLIOGRÁFICAS}

Bianchi, H. Ramification of the superficial branch of the radial nervus. Rev .Chil. Anat., 20(3):247-50, 2002.

Botte, M.; Cohen, M.; Lavernia, C.; Schroeder, H.; Gellman, H. \& Zinberg, E. The dorsal branch of the ulnar nerve: an anatomic study. J. Hand Surg., ??(5):603-7, 1990.

Casoli, V.; Vérolino, P.; Pélissier, P.; Kostopoulos, E.; Caix, P.; Delmas, V. \& Martin, D.; Baudet, J. The retrograde nerocutaneous island flap of the dorsal branch of the ulnar nerve: anatomical basis and clinical application. Surg. Radiol. Anat., 26(1):8-13, 2004.

Cavusoglu, T.; Ozden, H.; Comert, A.; Yazici, I.; Acar, H.; Tellioglu, A. \& Tekdemir, I. Topographical anatomy of the dorsal branch of the ulnar nerve and artery: a cadaver study. Surg. Radiol. Anat., 33(3):229-33, 2010.

Chatain, I. \& Bustamante, J. Anatomía macroscópica, funcional y clínica. México D. F. Sistemas Técnicos de Edición, 1986.

Ellis, H. Clinical Anatomy: applied anatomy for students and junior doctors. $11^{\mathrm{a}}$ ed. Massachusetts, Blackwell Publishing, 2006.

Goto, A.; Kunihiro, O.; Murase, T. \& Moritomo, H. The dorsal cutaneous branch of the ulnar nerve: an anatomical study. Hand Surg., 15(3):165-8, 2010.

Huanmanop, T.; Agthong, S.; Luengchwapong, K.; Sasiwongpakdee, T.; Burapasomboon, P. \& Chentanez, V. Anatomic characteristics and surgical implications of the superficial radial nerve. J. Med. Assoc. Thai., 90(7):1423-9, 2007.

Ikiz, A. \& Ücerler, H. Anatomic characteristics and clinical importance of the superficial branch of the radial nerve. Surg. Radiol. Anat., 26(6):453-8, 2004.

Klitscher, D.; Müller, L. \& Rommens, P. Anatomical course of the superficial branch of the radial nerve and clinical significance for surgical approaches in the distal forearm. Eur. J. Trauma. Emerg. Surg., 33(1):69-73, 2007.

Lama, P.; Potu, B. \& Bhat, K. High origin of dorsal branch of the ulnar nerve and variations in its branching pattern and distribution: a case report. Cases J., 2:9130, 2009.

Latarjet, M. \& Ruiz Liard, A. Anatomía humana. 4ª Ed. Buenos Aires, Médica Panamericana, 2004, V. 1.

Leversedge, F.; Goldfarb, C. \& Boyer, M. A Pocketbook manual of hand and upper extremity anatomy: Primus manus. Lippincott Williams \&Wilkins, 2010, 136p.

Llusá, M.; Merí, A \& Ruano, D. Manual y atlas fotográfico de anatomía del aparato locomotor. Buenos Aires, Panamericana, 2004.
Loukas, M.; Louis Jr., R.G.; Wartmann, C.T.; Tubbs, R.S.; Turan Ozdemir, S. \& Kramer, J. The clinical anatomy of the communications between the radial and ulnar nerves on the dorsal surface of the hand. Surg. Radiol. Anat., 30: 85-90, 2008.

Moore, K. \&Dalley, A. Anatomía con orientación clínica. $4^{\mathrm{a}}$ Ed. Buenos Aires: Médica panamericana, 2002. 1185 p.

Paul, S.; Das, S. \& Chaudhary, S. Higher origin of the dorsal branch of ulnar nerve and connection between it and the deep branch throughout the hypothenar muscles: a case report. Eur. J. Anat., 10(1):37-40, 2006.

Robson, A.; See, M.; Ellis, H. Applied anatomy of the superficial of the radial nerve. Clin. Anat.,21(1): 38-45, 2008.

Rouvière, H. \& Delmas, A. Anatomía humana: descriptiva, topográfica y funcional. 10 ${ }^{\mathrm{a}} \mathrm{Ed}$. Barcelona, Masson, 2002. V. 3.

Samarakoon, L.; Lakmal, K.; Thillainathan, S.; Bataduwaatachchi, V.; Anthony, D. \& Jayasekara, R. Anatomical relations of the superficial sensory branches of the radial nerve: a cadaveric study with clinical implications. Patient. Saf. Surg., 5(1):28-33, 2011.

Sinelnikov, R. Atlas de anatomía humana. $4^{\text {a }}$ Ed. Moscu, MIR, 1986, v. 3 .

Tellioglu, A.; Tekdemir, I.; Ersoy, M.; Karabag, O. Dorsal branches of superficial radial nerve: an anatomic study with potential clinical applications. Eur. J. Plast. Surg., 23(8):419-21, 2000.

Testut, L. \& Latarjet, A. Tratado de anatomía humana. $9^{\mathrm{a}}$ Ed. Barcelona: Salvat, 1972. V. 3.

Tryfonidis, M.; Charalambous, P.; Mills, S.; Jass, G.; Jacob, S.; Stanley, J. \& Hayton, J. Distal radial and ulnar landmarks used in percutaneous pin fixation: Anatomical relationship to the superficial radial and ulnar nerves. Hand Surg., 15(3):161-4, 2010.

Vergara-Amador, E. \& Nieto, J.L. Estudio anatómico de la rama superficial del nervio radial. Implicaciones quirúrgicas. Rev. Fac. Med., 58(3):214-20, 2010.

Williams, P. L.; Warwick, R.; Dyson, M. \& Bannister, L. H. Gray Anatomía. 37ª Ed. Guanabara-Koogan, Ed. Rio de Janeiro, 1995, v. 2.

Zaki, A. Anatomical study of the superficial branch of the radial nerve. Bull. Alex. Fac. Med.,42(2):525-30, 2006.

\section{Dirección para correspondencia: \\ Prof. Dr. Enrique Olave \\ Facultad de Medicina \\ Universidad de La Frontera \\ Temuco - CHILE \\ Email: enrique.olave@ufrontera.cl}

Recibido: 07-05-2013

Aceptado:19-08-2013 